ARTICLE

\title{
Systematic Uncertainty Due to Statistics in Monte Carlo Burnup Codes: Application to a Simple Benchmark with TRIPOLI-4-D
}

\author{
Emeric BRUN*, Eric DUMONTEIL and Fausto MALVAGI \\ Commissariat à l'Energie Atomique, Centre de Saclay, \\ Service d'Etude des Réacteurs et de Mathématiques Appliquées (DEN/DANS/DM2S/SERMA/LTSD), \\ F91191 Gif-sur-Yvette CEDEX, France
}

\begin{abstract}
A new burnup code, TRIPOLI-4-D, has been developed at CEA Saclay. It relies on the use of TRIPOLI-4 for the Monte Carlo neutron transport part, and on MENDEL nuclide depletion solver for the burnup calculation. Its main characteristics are pointwise energies, probability tables for the unresolved resonances range, potential fine multigroup calculation of reaction rates, the fourth order Runge-Kutta to solve Bateman's equations, predictor/corrector schemes, novice and expert levels (either launch the calculation with TRIPOLI-4 data set or potential C++ scripting to interact with the coupling macro, through the use of CINT, the ROOT C++ interpreter), post-processing with ROOT, possibility to perform parallel calculations, restart capability.

This code owns a special parameter that allows the user to calculate the statistical uncertainty on any physics quantity using the Central Limit Theorem (this very classical Monte Carlo method is also often referred to as "brute force" or "independent replicas" since it consists in replicating a given simulation many times using different random sequences). This technique is particularly adapted to massive parallelism, but can also be set up sequentially on personal computers. In this paper we will apply it on a simple case study, a Pressurized Water Reactor (PWR) cell, and we will analyze different quantities with their associated statistical error bars.

Comparing those results to reference simulations, we will try to highlight systematic errors, inherent to Monte Carlo burnup codes, and we will show that a particular systematic error, due to the number of simulated particles, has to be considered while performing Monte Carlo burnup simulations.
\end{abstract}

KEYWORDS: TRIPOLI-4-D, Monte Carlo, burnup, TRIPOLI-4, uncertainty propagation, parallelism

\section{Introduction}

Monte Carlo burnup codes are of quite recent interest for the neutronic community. Those codes are all based on a linking between Monte Carlo transport codes (e.g. MCNP, ${ }^{1)}$ MCNPX, ${ }^{2)}$ TRIPOLI-4 $\left.{ }^{3)} \ldots\right)$ and burnup capabilities $\left(\mathrm{ORIGEN}^{4)}\right.$ MENDEL $^{5}$...). Most of them are based on $\operatorname{MCNP}(\mathrm{X})$, like MONTEBURNS, ${ }^{6}$ MCNPX, ${ }^{7}$ ALEPH, ${ }^{8)}$ VESTA, ${ }^{9)}$ MURE $^{10)}$ but this year the first version of TRIPOLI-4-D ${ }^{11,12)}$ has been released.

Formally, all those codes solve the coupled Boltzmann/Bateman equations. Doing that with a proper Monte Carlo (MC) code would require to store all the isotopes and collision sites to be able to perform a true microscopic depletion of the fuel. Since this is obviously not possible (nowadays at least) this non linear problem is solved by successive iterations over a transport part (with fixed compositions) and a depletion part (with fixed fluxes or reaction rates). But doing so, systematic errors of a priori unknown magnitude can arise. Moreover, most codes do not propagate uncertainties (in fluxes, reaction rates and concentrations) from the first step to the last, but they limit themselves to providing step by step values.

In this paper we will first present the most general fea-

*Corresponding author, E-mail: emeric.brun@cea.fr

(c) 2011 Atomic Energy Society of Japan, All Rights Reserved. tures of TRIPOLI-4-D. We will then focus on the uncertainty sources (arising from both statistical and systematic errors) in Monte Carlo codes, emphasizing the treatment of statistical uncertainties in TRIPOLI-4-D, based on independent simulations thanks to its parallelism capabilities. Finally, we will define a simple but realistic benchmark (a Pressurized Water Reactor (PWR) cell). This benchmark will be studied to analyze a particular systematic error due to the number of neutrons, foreseen by Dumonteil and Diop ${ }^{13,14)}$ and by $\mathrm{Z}$. $\mathrm{Xu}$ et $a l^{15)}$ on simple analytical or numerical benchmark with ad hoc Monte Carlo codes.

In this paper we analyze and quantify the effect of the number of simulated neutrons on the final biais of the calculation. And this on a realistic case (a PWR pin cell) and with an industrial code (TRIPOLI-4-D). We show that it is something of worrying importance.

\section{TRIPOLI-4-D}

TRIPOLI-4-D is made of two C++ interfaces that wrap the functionalities of both TRIPOLI-4 and MENDEL. The first is a set of generic methods for MC neutrons transport, allowing to run a simulation, or to dynamically reload compositions or the geometry for example. The second provide methods to perform material depletion calculation with the fourth order Runge-Kutta method. Both interfaces are linked 
to the $\mathrm{C}++$ interpreter $\mathrm{CINT}^{16)}$ allowing to build advanced coupling schemes between transport and burnup which are either run interactively or compiled and executed with coupling scripts. This interpreter belongs to the $\operatorname{ROOT}^{17)}$ libraries developed at CERN and under LGPL public license. ROOT is linked to TRIPOLI-4-D offering numerous functionalities in terms of data treatment, analysis and visualisation.

From a user point of view its main characteristics are as follows:

- Use of CINT to launch transport/depletion sequences either interactively or in batch mode.

- Time irradiation performed with either fluxes or reaction rates, or both of them at the same time, on an isotope by isotope basis. In case fluxes are calculated, GENDF data (processed by NJOY ${ }^{18)}$ ) are used to calculate reaction rates afterward.

- Simulations with either a fixed flux, fixed source intensity or a fixed power defined by the user. In this last case, the power can be automatically calculated with TRIPOLI-4 deposited energy tally, or with provided kerma data.

- Three different time schemes: Euler explicit and two predictor-corrector (order 2) time schemes (midpoint and mean). Those two schemes are described in Ref. 19).

- Automatic statistical error propagation calculation using independent simulations.

- Full parallelisation with high scalability of the whole transport/depletion procedure.

- Use of ROOT and PROOF ${ }^{17)}$ (its parallelisation engine) to provide and speed up both the visualisation and the analysis of the results.

\section{Uncertainties Propagation in MC Burnup Codes}

Since Monte Carlo burnup codes make more assumptions than simple Monte Carlo transport codes, this section details the different sources of uncertainties inherent to burnup code, focusing on their treatment in TRIPOLI-4-D.

\section{Statistical Uncertainties}

In standard Monte Carlo transport codes, each calculated tally is provided together with an associated statistical uncertainty (the standard deviation of the probability density associated to the tally). In most Monte Carlo burnup codes, the coupling script iterates between a parallelized transport step and a mono-processed depletion step. At the end of the procedure, the statistical uncertainty of the transport step is thrown away and not propagated to the burnup step nor to the next transport. At the end, no confidence intervals are provided on isotopic inventories.

One of the solutions to propagate the statistical uncertainties through the different burnup steps is to perform independent simulations (often referred to as independent replicas or brute force ${ }^{20)}$ ) of this whole procedure: each transport/depletion iteration over all the time steps (each with a different sequence of random numbers) has to be re- peated many times, to be able to compute a probability distribution for each tally.

This procedure was implemented in TRIPOLI-4-D, which gives the user a reliable propagated statistical uncertainty. On top of that TRIPOLI-4-D uses this independent simulations mechanism to implement its parallelism ability; each processor handles its own set of independent MC burnup simulations, and the results are gathered at the end.

\section{Systematic Error}

Once the statistical uncertainties correctly computed, they can be used to detect systematic errors: the statistical uncertainty can indeed be decreased by increasing the number of simulated neutrons, allowing finding potentially small systematic effects. As explained in the introduction, Monte Carlo transport does very few assumptions, and the simulations are therefore often considered as reference ones because of this. Monte Carlo burnup codes are however really different, since at least three causes of systematic errors add to the statistical uncertainties:

- Fluxes and reaction rates are considered as stationary over the different step of the time scheme.

- Isotope densities are considered as uniform in each depletion volume/region (while in reality it is continuously varying over space).

- Bateman equation are non linear with respect to the fluxes and this induce a systematic error due to the number of neutrons (the lower the number of neutrons in each simulation, the bigger the bias).

This last bias has been extensively theoretically studied and can eventually be corrected. ${ }^{13,14)}$ It is caused by the fact that Bateman equation (that we call $g$ ) being non linear function of the flux, the real concentration of an isotope (called $N_{R}$ ) is a function of the real flux $\phi_{R}$ but differs from the expectation of Bateman equation solving with different estimated flux $\bar{\phi}$ :

$$
N_{R}=g\left(\phi_{R}\right) \neq E(g(\bar{\phi})) .
$$

In the following section of this paper we will try to quantify it with TRIPOLI-4-D on a very simple case study: a PWR cell.

\section{Application}

\section{Description of the Case Study}

This case study is a $4.9 \%$ enriched UOX PWR cell depleted until $60 \mathrm{GWd} / \mathrm{t}$. The fuel pin (Fig. 1) is divided into six annular depletion regions to take into account spatial flux distribution (spatial self-shielding effect). Reflection boundary conditions are attached to the 6 faces of the cell. JEFF3.1 nuclear data were used to run the simulations, with an optimized isotopic depletion chain made of 161 isotopes. For the sake of simplicity, the first order Euler depletion scheme was used. The irradiation period was divided into 45 steps. For each step, a 11,500 multigroup flux is estimated by TRIPOLI-4. From this flux and GENDF cross sections, reaction rates are built for each isotope except for U-238 which reaction rates are directly estimated by TRIPOLI-4 so 


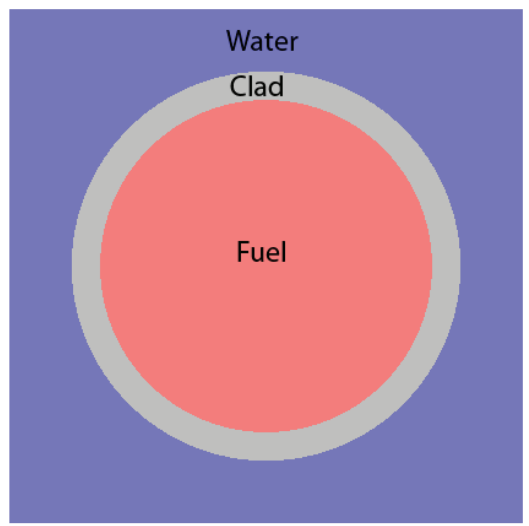

Fig. 1 Geometry of the PWR cell case study

as to properly capture self-shielding phenomenon.

To avoid source convergence problems while using a low number of neutrons, the simulations were performed in fixed source mode, with a uniformly distributed source. Two types of flux normalization were considered in this study: normalization to the power and fixed source intensity. The latter case was also studied to get rid of the normalization procedur used in the more realistic case of the normalization to the power since it may induce a bias propagating from step to step.

\section{Results}

To quantify the bias, different TRIPOLI-4-D simulations were run, each with a different number of neutrons per simulation (either $1 \times 1,5 \times 5,10 \times 10,20 \times 20,100 \times 100$, $100 \times 1,000$ or $1,000 \times 1,000$ neutrons, the first number being the number of cycles and the second the number of neutrons per cycle). However, the overall number of independent simulations in each case was adapted in order to have similar standard deviations.

The reason why such small numbers of neutrons are compared is that it provides a hint of what really happens when one simulate a full core. Indeed, when a full core of 100 axial zones and 200 assemblies is simulated, each being made of $17 \times 17$ pin cell, which are themselves made of 6 burnup regions, then a total number of roughly $10^{8}$ volumes have to be explored by the neutrons. With one billion neutrons for the whole simulation the average number of neutrons per volume is therefore of only 10. On top of that, this mean number even does not tell that some regions will have a good statistics of neutrons (e.g. outer rings of the pin cell) while some other will systematically see very few neutrons (e.g. inner zones), worsening the situation.

The following results are presented first for fixed power simulations, then for fixed source intensity. Each of these normalization methods induces a different expression of the renormalization factor $(\mathrm{RF})$ used to settle the flux and reaction rates level before depletion calculations.

(1) Fixed Power

In this case, a power level is specified by the user $\left(P_{\text {user }}\right)$. The flux level given by TRIPOLI-4 is arbitrary (it depends

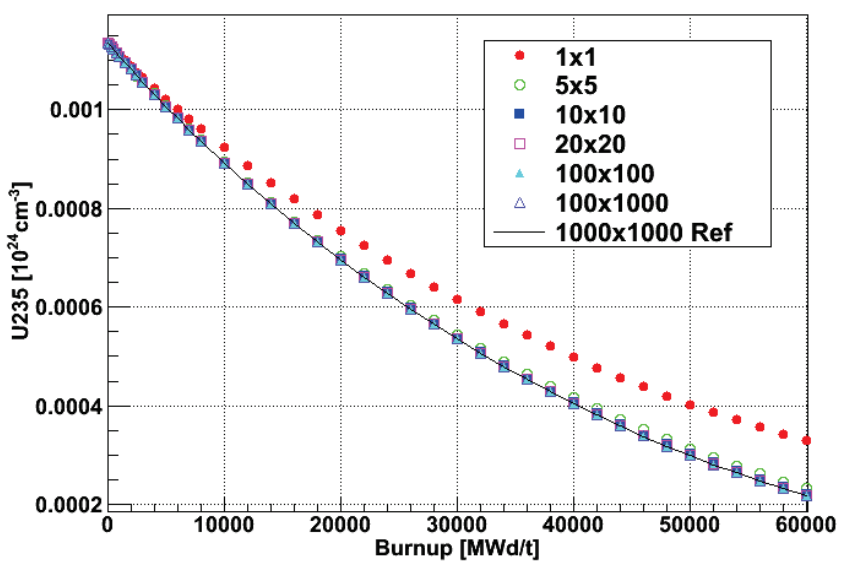

Fig. 2 Concentration of U-235 versus burn-up in the inner ring of the fuel cell

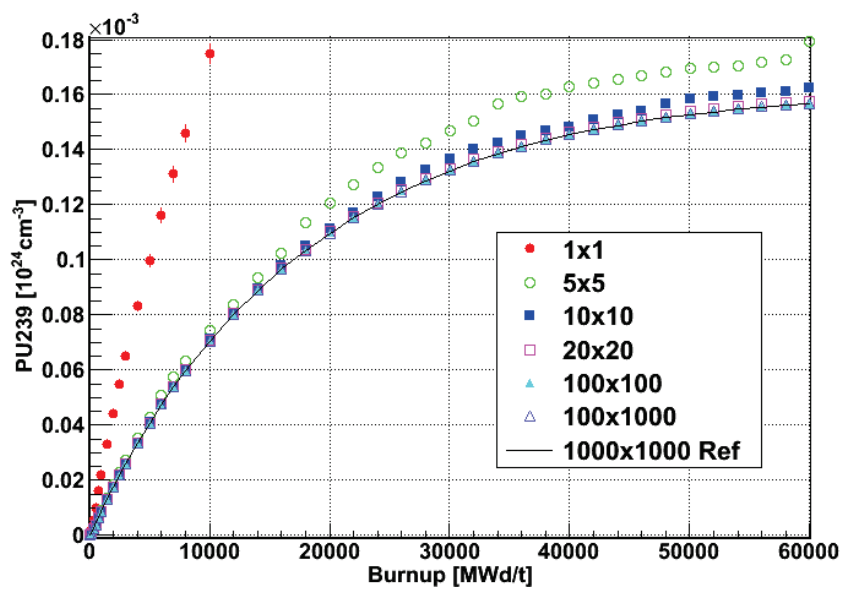

Fig. 3 Zoom on the concentration of $\mathrm{Pu}-239$ for the last burn-up steps in the inner ring of the fuel cell

essentially on the neutron source intensity) and corresponds to the power $P_{T 4}$ (calculated as the sum on all isotopes of the fission and capture rates multiplied by their corresponding energy release). The expression of RF is then:

$$
R F=P_{\text {user }} / P_{T 4}
$$

Ten independent simulations of the case with 1,000 cycles of 1,000 neutrons have been performed. Since this case should be the less biased and that statistical fluctuations between the 10 simulations remains negligible this case represents the reference. Figure 2 gives the U-235 concentration in the inner fuel cell as a function of the burnup for different number of neutrons per independent simulation. The one-sigma error bars are represented on top of the points but are small and do not appear at first sight. Only the $1 \times 1$ case seems to behave differently from the others. A detailed analysis of numerical values shows that the $5 \times 5,10 \times 10$ and $20 \times 20$ cases are also outside the statistical uncertainties, and biased of as much as $7 \%$.

Figure 3 gives also the concentration versus burnup, but for $\mathrm{Pu}-239$. $\mathrm{Pu}-239$ is produced while U-235 disappears, and therefore the $\mathrm{Pu}-239$ concentrations given by the different simulations are growing values of the burnup. Like U-235, 


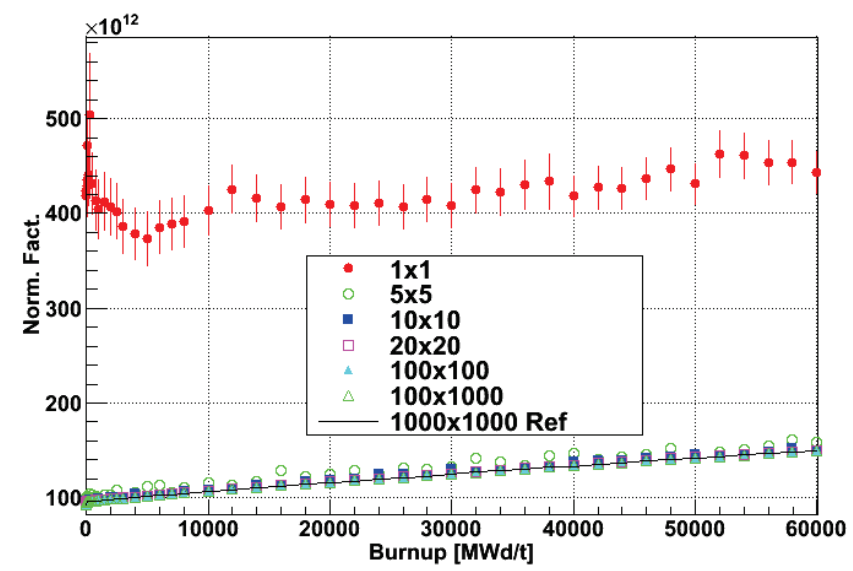

Fig. 4 Renormalization factor versus burn-up

this figure indicates that the bias is a decreasing function of the number of neutrons. The two last figures also show that the systematic error gets bigger with increased burnups. This is confirmed by a detailed numerical analysis that shows that for $\mathrm{Pu}-239$, in the case of $5 \times 5$ neutrons, the bias goes up to $15 \%$.

The mechanisms explaining the propagation and the amplification of this phenomenon from one step to the other do not rely only on the biased concentrations. Indeed, once the concentrations are biased different parameters can link one burnup step to the other, like the normalization factor, and become biased (Figs. 4 and 5). Obviously, once the concentrations are biased the transport sequences start from wrong material properties and the flux is also biaised (Fig. 6).

To characterize the bias more precisely Table 1 indicates for three selected families of isotopes ( $\mathrm{U}, \mathrm{Pu}$ and $\mathrm{Xe}$ ) both

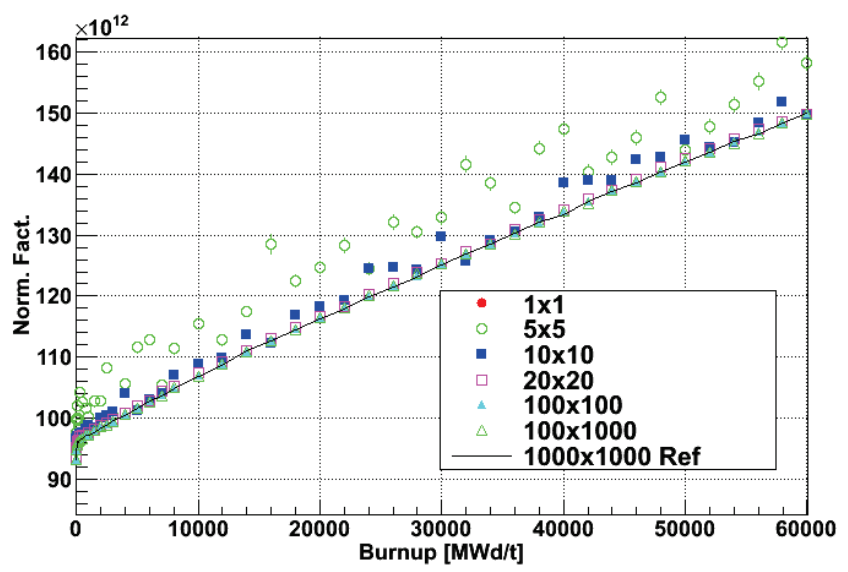

Fig. 5 Zoom on the renormalization factor versus burn-up

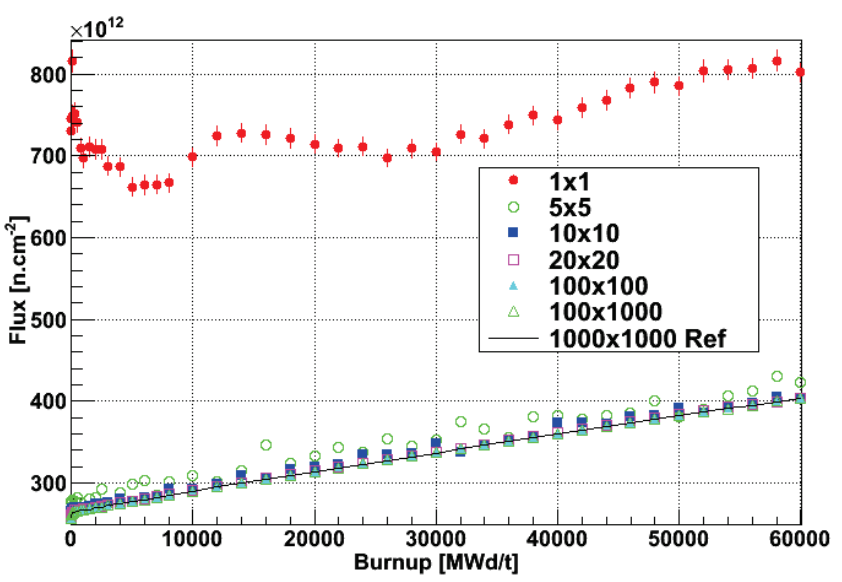

Fig. 6 Flux in the inner ring of the fuel cell versus burn-up

Table 1 Test of compatibility between selected isotopes concentration distributions and 100 independent replicas of $100 \times 1,000$ in the fixed power case. (Diff is the relative difference between means : Diff $=\left(\mathrm{m}-\mathrm{m}_{\mathrm{ref}}\right) / \mathrm{m}_{\mathrm{ref}} ; \mathrm{p}$ is the significance of the Student's $t$ test)

\begin{tabular}{lllllll}
\hline & \multicolumn{2}{c}{$5 * 5$} & \multicolumn{2}{c}{$20 * 20$} & \multicolumn{2}{c}{$100 * 100$} \\
\hline Isotope & Diff $\%$ & $p$ & \multicolumn{1}{c}{ Diff $\%$} & $p$ & Diff $\%$ & $p$ \\
\hline PU236 & 1.40 & 0.91 & -0.097 & 0.97 & 0.27 & 0.58 \\
\hline PU238 & -0.82 & 0.58 & 0.11 & 0.72 & -0.13 & 0.03 \\
\hline PU239 & $\mathbf{1 4 . 0 0}$ & $\mathbf{1 . e - 3 0}$ & 0.58 & 0.01 & 0.024 & 0.58 \\
\hline PU240 & $\mathbf{1 1 . 0 0}$ & $\mathbf{6 . e - 1 2}$ & -0.025 & 0.94 & -0.11 & 0.11 \\
\hline PU241 & $\mathbf{5 . 6 0}$ & $\mathbf{1 . e - 4}$ & 0.77 & 0.02 & 0.16 & 0.02 \\
\hline PU242 & $\mathbf{- 4 . 1 0}$ & $\mathbf{2 . e - 5}$ & -0.58 & 0.03 & $\mathbf{- 0 . 1 4}$ & $\mathbf{3 . e - 3}$ \\
\hline U232 & -0.07 & 0.99 & -0.88 & 0.68 & -0.98 & 0.02 \\
\hline U233 & 31.00 & 0.04 & 0.36 & 0.82 & -0.54 & 0.06 \\
\hline U234 & 4.90 & 0.03 & 0.10 & 0.79 & -0.20 & 0.005 \\
\hline U235 & $\mathbf{6 . 4 0}$ & $\mathbf{3 . e - 2 7}$ & $\mathbf{0 . 3 8}$ & $\mathbf{1 . e - 3}$ & 0.052 & 0.02 \\
\hline U236 & 0.38 & 0.30 & -0.055 & 0.47 & -0.027 & 0.05 \\
\hline U237 & 15.00 & 0.07 & -0.39 & 0.84 & -0.41 & 0.31 \\
\hline U238 & $\mathbf{- 0 . 1 9}$ & $\mathbf{1 . e - 2 7}$ & -0.0064 & 0.04 & $-8.6 e-4$ & 0.16 \\
\hline XE130 & $\mathbf{- 3 . 3 0}$ & $\mathbf{3 . e - 0 5}$ & -0.25 & 0.15 & -0.06 & 0.06 \\
\hline XE131 & -2.20 & 0.03 & -0.087 & 0.70 & 0.086 & 0.05 \\
\hline XE132 & 0.26 & 0.45 & -0.023 & 0.74 & -0.014 & 0.32 \\
\hline XE133 & 1.40 & 0.15 & -0.14 & 0.48 & -0.04 & 0.30 \\
\hline XE134 & $\mathbf{- 0 . 5 6}$ & $\mathbf{2 . e - 4}$ & -0.049 & 0.12 & $7.9 e-3$ & 0.21 \\
\hline XE135 & $\mathbf{1 5 . 0 0}$ & $\mathbf{3 . e - 1 3}$ & 0.79 & 0.06 & 0.16 & 0.04 \\
\hline & & & & & &
\end{tabular}




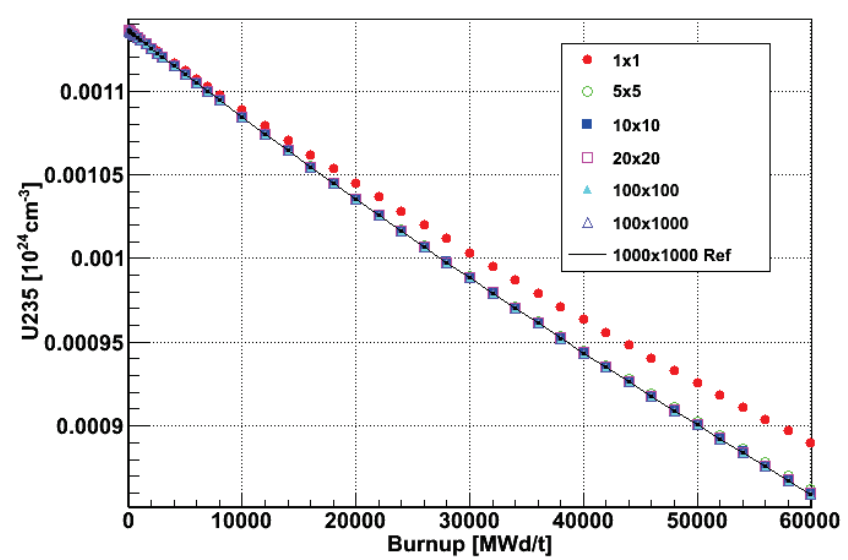

Fig. 7 Concentration of U-235 versus burn-up in the inner ring of the fuel cell

the difference in concentrations at the final burnup step for the inner ring between $5 \times 5,20 \times 20,100 \times 100$ neutrons simulations and 100 independent replicas of $100 \times 1,000$ (instead of the reference case $1,000 \times 1,000$ to ensure the robustness of the Student's $t$ test) and the significance $p$ of the Student's $t$ test. $^{21)}$ The significance $p$ is the probability that the difference between the means of two distributions could be this large just by chance for distributions with equal means. Therefore, a small significance $(0.05$ or 0.01$)$ means that the observed difference is statistically very significant. The results below $1 \%$ probability are indicated in bold, and red. As one can see, in the $5 \times 5$ case, half of the chosen isotopes concentrations differ from the reference with pretty important differences. For the $20 \times 20$ case this number falls to two isotopes over the 20 considered (U-235 and Xe-136), while only one remains different in the $100 \times 100$ case $(\mathrm{Pu}-242)$. All those observations clearly tell that there is a bias depending on the number of neutrons in each independent simulation.

(2) Fixed Source Intensity

In this case, the flux level is imposed by the neutron source intensity defined by the user. Thus, $\mathrm{RF}=1$.

In the case of fixed source intensity almost no other parameters than concentrations couple a burnup step to the next one. As one can see on the same figures studied before, the bias stays roughly unchanged with respect to the fixed power simulations (Figs. 7 to 9 and Table 2). Table 2 even indicates that the results are worse in the fixed source intensity case. Indeed, the comparison of simulations with $5 \times 5,20 \times 20$ and $100 \times 100$ neutrons to the reference case shows that 7,9 and 2 isotopes respectively over 20 fail to the Student's $t$ test. These numbers can be compared to 10, 2 and 1 isotope over 20 that fail to this test in the case of fixed power. One can conclude that the calculation bias showed by simulations with an insufficient number of particles is really linked to the burn-up calculation, and not to the normalization procedure.

\section{Conclusion}

This paper aimed at giving a brief overview on uncertainties treatment in MC burnup code, and in particular in TRIPOLI-4-D. Two different kinds of uncertainties have to

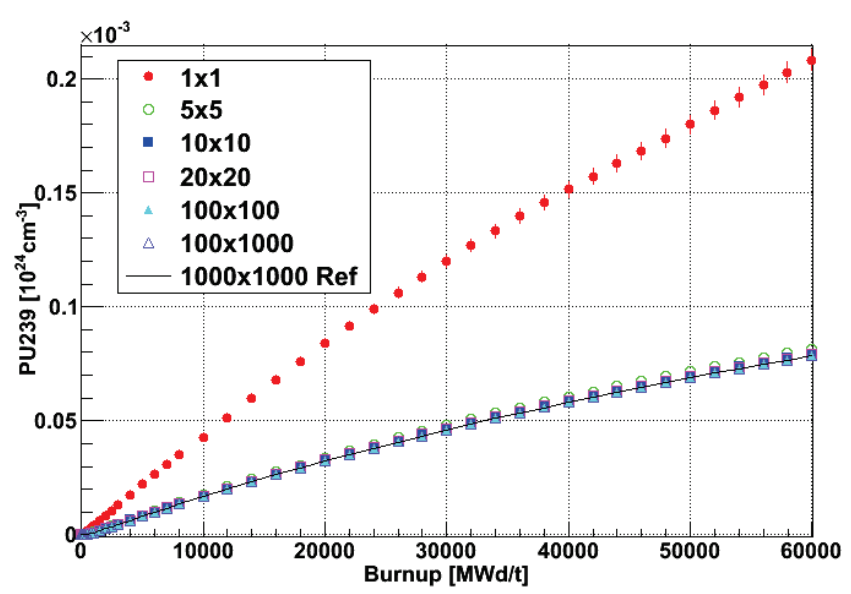

Fig. 8 Concentration of $\mathrm{Pu}-239$ versus burn-up in the inner ring of the fuel cell

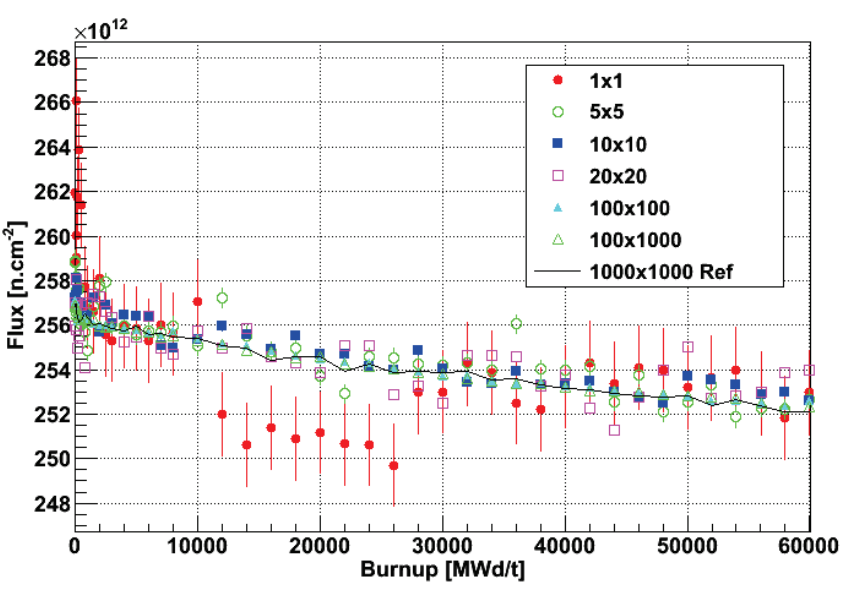

Fig. 9 Flux in the inner ring of the fuel cell versus burn-up

be taken into account, and propagated through the burnup steps. Statistical uncertainties (propagation) have to be taken into account in MC burnup code, and the formal approach to deal with them is by using independent replicas. This was implemented in TRIPOLI-4-D and allowed to work on the systematic uncertainties in burnup codes. Those may arise from at least three sources: the discretization in time, in space, or in number of neutrons (i.e. the time/burnup step structure, the spatial binning, and the number of neutrons). This last source of systematic errors has been studied on a PWR cell with TRIPOLI-4-D. It has been shown that there was indeed a potentially strong bias depending on the number of neutrons in independent simulations.

This bias has to be dealt with because it may affect the whole burning procedure in case the depletion regions have too few neutrons. This may happen in case of full core modelling, because to take into account the spatial gradient of the flux, the core has to be studied on a pin by pin basis. If one considers 6 depletion volumes in each pin cell, $17 \times 17$ pin cells per assembly, roughly 200 assemblies in the core and 100 axial zones, then even for $100 \times 100$ neutrons per volume the total number of simulated neutrons should be of at least $10^{12}$ to get rid of the effect. If the brute force approach is used to treat statistical uncertainties, the user have to make sure that not only the overall number of neutrons is big 
Table 2 Test of compatibility between selected isotopes concentration distributions and 100 independent replicas of $100 \times 1000$ in the fixed source intensity case. (Diff is the relative difference between means : Diff $=\left(\mathrm{m}-\mathrm{m}_{\mathrm{ref}}\right) / \mathrm{m}_{\mathrm{ref}} ; \mathrm{p}$ is the significance of the Student's $t$ test)

\begin{tabular}{lcccccc}
\hline & \multicolumn{2}{c}{$5 * 5$} & \multicolumn{2}{c}{$20 * 20$} & \multicolumn{2}{c}{$100 * 100$} \\
\hline Isotope & Diff $\%$ & $p$ & Diff $\%$ & Isotope & Diff \% & $p$ \\
\hline PU236 & 68.00 & 0.22 & -2.60 & 0.29 & 0.74 & 0.12 \\
\hline PU238 & 3.00 & 0.27 & 0.70 & 0.11 & $\mathbf{0 . 3 9}$ & $\mathbf{1 . e - 4}$ \\
\hline PU239 & $\mathbf{3 . 7 0}$ & $\mathbf{2 . e - 7}$ & $\mathbf{0 . 5 3}$ & $\mathbf{4 . e - 6}$ & -0.021 & 0.31 \\
\hline PU240 & $\mathbf{4 . 7 0}$ & $\mathbf{3 . e - 5}$ & $\mathbf{0 . 8 6}$ & $\mathbf{1 . e - 6}$ & 0.062 & 0.11 \\
\hline PU241 & -3.30 & 0.06 & $\mathbf{0 . 7 9}$ & $\mathbf{8 . e - 3}$ & $-\mathbf{0 . 1 9}$ & $\mathbf{0 . 0 0 3}$ \\
\hline PU242 & $\mathbf{- 5 . 1 0}$ & $\mathbf{1 . e - 3}$ & $\mathbf{1 . 2 0}$ & $\mathbf{1 . e - 4}$ & -0.087 & 0.11 \\
\hline U232 & 90.00 & 0.13 & -3.00 & 0.22 & 0.51 & 0.23 \\
\hline U233 & $1.1 \mathrm{e}+2$ & 0.02 & -1.30 & 0.47 & 0.39 & 0.22 \\
\hline U234 & 2.90 & 0.48 & -0.41 & 0.52 & 0.12 & 0.27 \\
\hline U235 & $\mathbf{0 . 3 1}$ & $\mathbf{7 . 9 e - 1 1}$ & $\mathbf{0 . 0 4 8}$ & $\mathbf{7 . e - 1 1}$ & $-1.5 e-3$ & 0.34 \\
\hline U236 & 0.085 & 0.71 & $5.4 e-3$ & 0.87 & $-4.2 e-5$ & 0.99 \\
\hline U237 & -0.33 & 0.96 & -0.06 & 0.97 & 0.40 & 0.2 \\
\hline U238 & $\mathbf{- 0 . 0 2 1}$ & $\mathbf{1 . e - 0 9}$ & $\mathbf{- 3 . 7 e - 3}$ & $\mathbf{3 . e - 1 1}$ & $6.7 \mathrm{e}-5$ & 0.52 \\
\hline XE130 & -0.076 & 0.93 & $\mathbf{- 0 . 4 7}$ & $\mathbf{2 . e - 4}$ & 0.023 & 0.41 \\
\hline XE131 & -0.42 & 0.12 & -0.018 & 0.75 & 0.022 & 0.02 \\
\hline XE132 & -0.43 & 0.03 & -0.039 & 0.25 & -0.011 & 0.06 \\
\hline XE133 & -0.48 & 0.77 & $8.3 e-3$ & 0.98 & 0.13 & 0.09 \\
\hline XE134 & $\mathbf{- 0 . 6 4}$ & $\mathbf{1 . e - 5}$ & $\mathbf{- 0 . 0 6 1}$ & $\mathbf{0 . 0 0 7}$ & 0.0029 & 0.53 \\
\hline XE135 & 0.58 & 0.53 & $-6.3 e-3$ & 0.97 & $-2.4 e-3$ & 0.95 \\
\hline XE136 & $\mathbf{- 1 . 8 0}$ & $\mathbf{1 . e - 1 6}$ & $\mathbf{- 0 . 1 8}$ & $\mathbf{2 . e - 0 6}$ & $5.5 e-3$ & 0.45 \\
\hline
\end{tabular}

enough, but also the number of neutrons per independent simulations.

In all cases, the natural scale for fixing the number of neutrons is the uncertainty on nuclear data (which are not specific to MC burnup code but to MC transport codes more generally). Those uncertainties may affect the results of several percent on final quantities like concentration, reaction rates or fluxes.

Further developments concerning TRIPOLI-4-D will concern the treatment of this bias using recently proposed methods, ${ }^{13,14)}$ and the quantification of other systematic errors (e.g. the smooth variation of macroscopic cross sections).

\section{Acknowledgment}

The authors want to acknowledge EDF and AREVA for partial financial support, as well as C.M. Diop and A. Zoia for their fruitful advises.

\section{References}

1) X-5 Monte Carlo Team, MCNP - A General Monte Carlo N-Particle Transport Code, Version 5, LA-UR-03-1987, Los Alamos National Laboratory (LANL) (2003).

2) J. S. Hendricks et al., MCNPX 2.6.0 Extensions, LA-UR-08-2216, Los Alamos National Laboratory (LANL) (2008).

3) C. M. Diop, E. Dumonteil, F. X. Hugot, C. Jouanne, Y. K. Lee, F. Malvagi, A. Mazzolo, O. Petit, J. C. Trama, "An Overview on the Monte Carlo Particle Transport Code TRIPOLI-4," Trans. Am. Nucl. Soc., 97, 694-695 (2007).
4) M. J. Bell, ORIGEN - The ORNL Isotope Generation and Depletion Code, ORNL-4628 UC-32, Oak Ridge National Laboratory (ORNL) (1973).

5) A. Tsilanizara, S. Lahaye, E. Brun, CEA Internal Report, (2009)

6) H. R. Trellue, Development of Monteburns: a code that links MCNP and ORIGEN2 in an automated fashion for burnup calculations, LA-13514-T, Los Alamos National Laboratory (LANL) (1998).

7) M. L. FENSIN, J. S. Hendricks, S. Anghaie, "The Enhancements and Testing of the MCNPX Depletion Capability," 2008 International Congress on Advances in Nuclear Power Plants, Anaheim, CA, (2008).

8) W. Haeck, B. Verboomen, "An optimal approach to Monte Carlo Burnup,” Nucl. Sci. Eng., 156, 180-196 (2007).

9) W. Haeck, VESTA User's manual, IRSN DSU/SEC/T/2008-331 Indice A (2008).

10) O. Meplan et al. "MURE : MCNP Utility for Reactor Evolution - Description of the method, first applications and results," ENC2005 European Nuclear Conference. Nuclear Power for the XXIst Century: From basics research to high-tech industry, Versailles, France (2005).

11) E. Brun, E. Dumonteil, CEA Internal report (2010).

12) R. Reyes-Ramírez, C. Martín-del-Campo, J.-L. François, E. Brun, E. Dumonteil, F. Malvagi, "Comparison of MCNPX-C90 and TRIPOLI-4-D for fuel depletion calculations of a Gas-cooled Fast Reactor," Ann. Nucl. Energy, 37, 1101-1106 (2010).

13) E. Dumonteil, C. M. B. Diop, "Unbiased minimum variance estimator of a matrix exponential function. Application to Boltzmann/Bateman coupled equations solving," Proc. Int. Conf. on Mathematics Computational Methods \& Reactor Physics, New York, May 3-7, 2009 (2009). 
14) E. Dumonteil, C. M. Diop, "Biases and error bars in Monte Carlo burnup calculations: an unbiased stochastic scheme to solve Boltzmann/Bateman coupled equations," Nucl. Sci. Eng., 167, 165-170 (2011)

15) Z. Xu, J. Rhodes, K. Smith, "Statistical implications in Monte Carlo depletions," Proc. of PHYSOR2010, Pittsburgh, Pennsylvania, USA, May 9-14, 2010 (2010).

16) M. Goto, "C++ interpreter - cint," CQ publishing (1997).

17) I. Antcheva, M. Ballintijn, B. Bellenot et al., "ROOT - A C++ framework for petabyte data storage, statistical analysis and visualization," Comp. Phys. Comm., 180, 2499-2512 (2009).

18) R. E. MacFarlane, D. W. Muir, The NJOY Nuclear Data Processing System Version 91, LA-12740, Los Alamos Na- tional Laboratory (LANL) (1994).

19) M. L. Fensin, J. Hendricks, H. Trellue, S. Anghaie, Incorporation of a Predictor-Corrector Methodology and 1-group reaction rate reporting scheme for the MCNPX depletion capability, LA-UR-06-3925, Los Alamos National Laboratory (LANL); Trans. Am. Nucl. Soc., 95, 317 (2006).

20) N. Garcia-Herranz et al., "Propagation of statistical and nuclear data uncertainties in Monte Carlo burnu-up calculations," Ann. Nucl. Energy, 35, 714-730 (2008).

21) W. H. Press, S. A. Teukolsky, W. T. Vetterling, B. P. Flannery, Numerical Recipes in C, Second Edition, Cambridge University Press, (1992). 